\title{
A EFICIÊNCIA RELATIVA DOS PORTOS DO SUL DO BRASIL NO TRANSBORDO DE GRANÉIS SÓLIDOS
}

\author{
Sandra Regina da Silva Pinela \\ Universidade Tecnológica Federal do Paraná \\ Rua Cristo Rei n $^{\circ} 19$ - Vila Becker, Toledo - PR \\ sandrapinela@utfpr.edu.br \\ Weimar Freire da Rocha Júnior \\ Universidade Estadual do Oeste do Paraná \\ Rua da Faculdade n ${ }^{\circ} 645$ - Jardim Santa Maria, Toledo - PR \\ wrochajr2000@gmail.com \\ Marcos Roberto Bombacini \\ Universidade Tecnológica Federal do Paraná \\ Rua Cristo Rei no 19 - Vila Becker, Toledo - PR \\ bombacini@utfpr.edu.br \\ Tatiani Sobrinho Del Bianco \\ Universidade Tecnológica Federal do Paraná \\ Rua Cristo Rei n $^{\circ} 19$ - Vila Becker, Toledo - PR \\ tatiani.sdelbianco@gmail.com

\section{RESUMO}

Este artigo tem como objetivo analisar a eficiência técnica dos portos marítimos do Sul do Brasil por meio da Análise Envoltória de Dados - DEA, com enfoque no transbordo de granéis sólidos na faixa do cais. O período analisado foi de 2010 a 2016 e os dados, coletados por meio de pesquisa de campo e documental. A escolha das variáveis do modelo matemático se deu pelo método de Análise dos Componentes Principais. Os resultados mostraram que os portos são ineficientes quanto à rapidez do transbordo, o que afeta o critério confiabilidade na escolha de serviços pelos clientes.

Palavra-chave: Logística; Eficiência; Fronteira de Ineficiência; Portos marítimos; Granéis sólidos.

\begin{abstract}
This paper investigates the technical efficiency of different Brazilian South ports using Data Envelopment Analysis - DEA, focusing on the transshipment of solid bulk terminal in the quay mooring zone. The study was conducted between 2010 and 2016 and the report was based on desk and field research. Principal Components Analysis was used as a tool in the mathematical model variables selection. The results revealed low efficiency related to the transshipment operation affects customers criterion reliability.
\end{abstract}

Keywords: Logistics; Efficiency; Border of Inefficiency; Seaports; Solid bulk. 


\section{Como Citar:}

PINELA, S. R. S.; et al. A Eficiência Relativa dos Portos do Sul do Brasil no Transbordo de Granéis Sólidos. In: SIMPÓSIO DE PESQUISA OPERACIONAL E LOGÍSTICA DA MARINHA, 19., 2019, Rio de Janeiro, RJ. Anais [...]. Rio de Janeiro: Centro de Análises de Sistemas Navais, 2019.

\section{INTRODUÇÃO}

O objetivo desse estudo foi analisar a eficiência técnica dos portos marítimos do sul do Brasil pela Análise Envoltória de Dados - DEA e a aplicação do método se deu pela abordagem Variable Returns to Scale (VRS) ou BCC (Banker, Charnes e Cooper, 1984), com enfoque no transbordo de granéis sólidos na faixa do cais, uma vez que o Brasil têm participado ativamente com esse produto nas exportações para o comércio internacional.

De acordo com a OMC (2013), entre 1980 e 2011, o crescimento médio obtido nessas transações foi de $7 \%$ a. a. e os países em desenvolvimento elevaram suas participações de $34 \%$ para $47 \%$, cujo volume, ao longo deste período, representou duas vezes a produção mundial. No período de 2010 a 2016, as exportações brasileiras corresponderam a $24,7 \%$ do Produto Interno Bruto (PIB) a preços correntes (BRASIL, 2017). Segundo a Conab (2016), na safra de 2014/2015 a soja representou $45 \%$ do total da produção de grãos do país, que geram volumes de exportação significativos nos portos do Sul do Brasil (MDIC, 2016).

Apesar de ser altamente dependente desses entrepostos logísticos para realizar as suas exportações, segundo Rios (2005), Fontes (2006), Wanke, Barbastefano e Hijjar (2011), Peixoto (2013), Martins (2015), Rios (2015) e Pires (2016), os portos brasileiros demonstraram ser ineficientes por uma série de fatores.

Assim, os pressupostos a serem validados neste estudo se orientam sob a perspectiva econômica de que um porto eficiente é aquele capaz de produzir mais embarques e desembarques, em determinado período de tempo, sem empregar unidades adicionais de recursos disponíveis. Nesse sentido, a eficiência está relacionada com a produtividade.

Diante o exposto, o estudo foi estruturado em cinco seções, sendo a primeira esta introdução, que faz considerações gerais sobre o assunto. A segunda se refere à fundamentação teórica sobre o tema. Na terceira seção são apresentados os procedimentos metodológicos e, a seguir, se faz a análise e discussão dos resultados. Por fim, apresenta-se a conclusão.

\section{PROCEDIMENTOS METODOLÓGICOS}

Esta pesquisa se caracteriza como explicativa, em que os portos marítimos do Brasil representam o universo e os portos do Sul a amostra, definida como não probabilística, por tipicidade (VERGARA, 2004). O transbordo na faixa do cais foi considerado o objeto de análise, por se tratar de uma estrutura, que segundo Wanke e Hijjar (2009) está organizada para a transferência de cargas.

Os granéis sólidos compuseram o tipo de carga a ser analisado, pois segundo Menegazzo e Fachinello (2014), são os mais movimentados nos portos brasileiros. Os indicadores de desempenho foram constituídos a partir da quantidade de atracações que o porto realiza, o tempo atracado e o peso das cargas. Os dados foram coletados entre 22/04/2017 e 01/06/2017, disponibilizados pelo Sistema de Informações Gerenciais (SIG) da Agência Nacional de Transportes Aquaviários (ANTAQ, 2017).

Dos nove portos marítimos instalados na região Sul (24\% do total do sistema portuário nacional), sete foram selecionados por movimentarem granéis sólidos no período 
de análise, sendo eles: Porto de Paranaguá (PR), Porto de Antonina (PR), Porto de São Francisco do Sul (SC), Porto de Imbituba (SC), Porto do Rio Grande (RS), Porto de Porto Alegre (RS) e Porto de Pelotas (RS).

A abordagem de corte transversal pareceu bastante adequada, devido à limitação temporal dos dados disponíveis (somente a partir de 2010), e ao uso satisfatório dessa estratégia em trabalhos sobre eficiência portuária, como os de Barros (2003), Cullinane e Wang (2006), Nwanosike, Nicoleta e Warnock-Smith (2012). Complementarmente, as informações sobre a infraestrutura dos portos foram extraídas dos Planos Mestres, elaborados e disponibilizados pela Secretaria dos Portos da Presidência da República (SEP/PR), e dos endereços eletrônicos das instalações portuárias.

Para validar os dados secundários, no mês de agosto de 2016 foram aplicadas entrevistas estruturadas aos gestores de logística dos portos de Paranaguá e São Francisco do Sul. Durante os meses de agosto e setembro de 2016, se realizou visitas técnicas nos portos de Paranaguá e São Francisco do Sul. No primeiro, foram observadas as seguintes instalações: administrativas, cais público, armazéns, silos, terminal privado de contêineres e pátios de armazenagem. No segundo, se observou o transbordo no cais público, os equipamentos portuários e de pesagem. Os processos-chaves do estudo consistem em: recebimento, armazenagem e carregamento de granéis sólidos.

O método de análise foi o Data Envelopment Analysis (DEA), desenvolvido por Charnes, Cooper e Rhodes (1978) e estendido por Banker, Charnes e Cooper (1984). Essa técnica admite a medida de eficiência produtiva de Farrel (1957) e a generalização da eficiência de Koopmans (1951), sob a ideia de fronteira de Pareto. Por meio dessa técnica é possível converter múltiplos insumos e produtos em uma única medida de eficiência e comparar organizações complexas, sob enfoque das melhores práticas.

Os requisitos levados em conta para aplicar o método consistem em: os portos realizarem as mesmas tarefas, apresentarem objetivos semelhantes, operaram nas mesmas condições de mercado e os inputs e output serem iguais, exceto em medida e escala (CHARNES, COOPER e RHODES, 1978).

Conforme Charnes, Cooper e Rhodes (1978), a unidade fundamental de análise do método DEA é denominada de Decision Making Units - DMU, que neste estudo são representadas pelos portos da região Sul do Brasil e, devido às atividades de transbordo no cais operarem sob o conceito de eficiência dos inputs, a abordagem DEA-BCC pareceu bastante adequada. Além disso, segundo Guerreiro (2006), ela admite competição imperfeita, o que faz coerente a explicação de Cullinane e Wang (2006) sobre ser a mais realista por incluir como eficientes DMUs não captadas pela reta construída no modelo de ganhos constantes de escala, ou CCR, o que torna mais benevolente a abordagem BCC.

Para o problema de Programação Linear (PL), o conceito de dualidade foi considerado. O modelo dual (Envelope), descrito por Roll e Hayuth (1993), permite fazer comparação entre as DMUs. Segundo Charnes, Cooper e Rhodes (1978), o resultado será a máxima proporção de saídas e entradas ponderadas, sujeitas à condição de que as proporções similares de cada DMU sejam menores ou iguais à unidade. Assim, o modelo se configurou na Equação 1, onde: $\mathrm{E}_{0}$ é a variável que representa a eficiência da $\mathrm{DMU}_{0} ; v_{i}$ e $u_{j}$ são os pesos dos inputs $i, i=1, \ldots, r$, e outputs $j, j=1, \ldots, s$, respectivamente; $x_{i k}$ e $y_{j k}$ são inputs $i$ e outputs $j$ da $\mathrm{DMU}_{k}, k=1, \ldots, n ; x_{i 0}$ e $y_{j 0}$ são os inputs $i$ e outputs $j$ da $\mathrm{DMU}_{0}$.

$$
\operatorname{Max} E_{0}=\sum_{j=1}^{s} u_{j} y_{j 0}+u^{*}
$$

Sujeito a 


$$
\begin{aligned}
& \sum_{i=1}^{r} v_{i} x_{i 0}=1 \\
& -\sum_{\substack{r \\
v_{i}}} v_{i} x_{i k}+\sum_{j=1}^{s} u_{j} y_{j k}+u^{*} \leq 0, u^{*} \in \mathcal{R}
\end{aligned}
$$

De acordo com Angulo Meza et al. (2003), o resultado da simulação testa a robustez dos valores, porém, os resultados podem oferecer perspectivas antagônicas em relação à eficiência. Por isso, além da análise da fronteira de eficiência, que representa a visão otimista, calculou-se a fronteira invertida, pela troca dos inputs por outputs do modelo original. Esse procedimento evitou que alguma DMU utilizasse somente variáveis que fossem mais favoráveis para o alcance da máxima eficiência. Para Soares de Melo et al. (2005), essa fronteira é composta pelas DMUs com as piores práticas gerenciais e, por isso, denominadas de ineficiente.

O confronto das duas perspectivas possibilitou identificar as DMUs sem relação essencial entre elas ou "falsamente" eficientes (ENTANI, MAEDA e TANAKA, 2002). Esse diagnóstico é feito pelo cálculo da eficiência composta, que, segundo Soares de Melo et al. (2005), consiste na média da diferença entre a eficiência padrão e a eficiência invertida. Depois disso, o resultado foi normalizado pelo cálculo da razão entre a eficiência padrão de cada DMU e o maior índice dentre todas as DMUs (MEZA et al, 2005), no sentido de identificar as unidades que efetivamente foram eficientes.

\subsection{DEFINIÇÃO DAS VARIÁVEIS DO MÉTODO DEA}

A seleção das variáveis se basearam nos trabalhos de Roll e Rayuth (1993), Barros (2003), Rios (2005), Cullinane e Wang (2006), Wanke, Barbastefano e Hijjar (2011), Sousa Júnior et al. (2013), Rios (2015), Wanke e Barros (2015) e foram validadas pelos gestores de logística nas entrevistas.

A quantidade de atracações representou o output do modelo DEA, definido como o limite final do fluxo de entrega nos portos. As variáveis de input foram constituídas pelo peso (em toneladas) e o tempo de operação das cargas, demonstrados na Tabela 1.

\begin{tabular}{|c|c|c|c|c|c|c|c|c|c|}
\hline \multirow[b]{2}{*}{ Porto } & \multirow[b]{2}{*}{ Ano } & \multirow[b]{2}{*}{$\begin{array}{c}\mathrm{N}^{\mathrm{o}} \\
\text { Atracações }\end{array}$} & \multirow[b]{2}{*}{$\begin{array}{l}\text { Carga Bruta } \\
(\mathrm{em} 1.000 \mathrm{t})\end{array}$} & \multicolumn{6}{|c|}{ Tempo (em $1.000 \mathrm{~h})$} \\
\hline & & & & Operação & $\begin{array}{c}\text { Navio } \\
\text { Atracado }\end{array}$ & Estadia & $\begin{array}{l}\text { Espera } \\
\text { Atracar }\end{array}$ & $\begin{array}{c}\text { Espera } \\
\text { Iniciar } \\
\text { Operação }\end{array}$ & $\begin{array}{c}\text { Espera } \\
\text { Desatracar }\end{array}$ \\
\hline \multirow{7}{*}{ 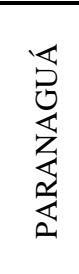 } & 2010 & 861 & $23.811,64$ & 64,7 & 78,05 & 322,78 & 244,73 & 0,68 & 0,74 \\
\hline & 2011 & 841 & $26.735,60$ & 72,67 & 88,48 & 591,63 & 503,15 & 1,21 & 0,74 \\
\hline & 2012 & 922 & $29.874,84$ & 73,65 & 96,68 & 787,67 & 690,98 & 0,3 & 0,85 \\
\hline & 2013 & 896 & $30.149,85$ & 75,66 & 95,93 & $1.084,59$ & 988,66 & 0,77 & 1,38 \\
\hline & 2014 & 813 & $29.596,19$ & 63,57 & 81,73 & 539,32 & 457,59 & 2,9 & 3,32 \\
\hline & 2015 & 742 & $29.475,77$ & 64,06 & 213,11 & $1.910,13$ & $1.697,02$ & 8,63 & 7,32 \\
\hline & 2016 & 775 & $28.235,58$ & 59,74 & 521,26 & $5.712,29$ & $5.191,04$ & 20,96 & 26,26 \\
\hline \multirow{7}{*}{ 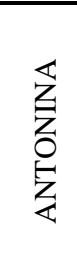 } & 2010 & 18 & 185,58 & 2,76 & 3,1 & 6,82 & 3,72 & 0,14 & 0,2 \\
\hline & 2011 & 84 & $1.102,63$ & 9,67 & 11,99 & 44,05 & 32,06 & 0,34 & 0,59 \\
\hline & 2012 & 82 & $1.161,55$ & 7,73 & 9,24 & 46,47 & 37,23 & 0,16 & 0,6 \\
\hline & 2013 & 103 & $1.537,21$ & 11,01 & 13,48 & 48,49 & 35,01 & 0,37 & 0,94 \\
\hline & 2014 & 97 & $1.472,86$ & 9,45 & 12,5 & 25,84 & 13,34 & 0,27 & 0,92 \\
\hline & 2015 & 46 & 761,06 & 6,67 & 17,21 & 57,4 & 40,19 & 0,65 & 1,84 \\
\hline & 2016 & 54 & $1.181,50$ & 9,12 & 43,8 & 80,81 & 37,01 & 1,37 & 2,31 \\
\hline
\end{tabular}

Tabela 1- Dados operacionais dos portos do Sul do Brasil para transbordo de granéis sólidos 2010 a 2016. 


\begin{tabular}{|c|c|c|c|c|c|c|c|c|c|}
\hline \multirow[b]{2}{*}{ Porto } & \multirow[b]{2}{*}{ Ano } & \multirow[b]{2}{*}{$\begin{array}{c}\mathrm{N}^{\mathbf{o}} \\
\text { Atracações }\end{array}$} & \multirow[b]{2}{*}{$\begin{array}{l}\text { Carga Bruta } \\
(\mathrm{em} 1.000 \mathrm{t})\end{array}$} & \multicolumn{6}{|c|}{ Tempo (em $1.000 \mathrm{~h})$} \\
\hline & & & & Operação & $\begin{array}{c}\text { Navio } \\
\text { Atracado }\end{array}$ & Estadia & $\begin{array}{l}\text { Espera } \\
\text { Atracar }\end{array}$ & $\begin{array}{c}\text { Espera } \\
\text { Iniciar } \\
\text { Operação }\end{array}$ & $\begin{array}{c}\text { Espera } \\
\text { Desatracar }\end{array}$ \\
\hline \multirow{7}{*}{ 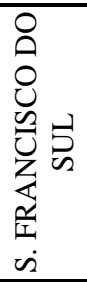 } & 2010 & 128 & $5.282,94$ & 8,45 & 10,26 & 48,02 & 37,76 & 0,16 & 0,15 \\
\hline & 2011 & 171 & $5.369,03$ & 10,62 & 13,96 & 53,6 & 39,65 & 0,28 & 0,61 \\
\hline & 2012 & 177 & $7.011,81$ & 10,43 & 15,06 & 51,96 & 36,9 & 0,44 & 2,01 \\
\hline & 2013 & 232 & $9.318,50$ & 13,99 & 15,09 & 67,78 & 52,68 & 0,25 & 0,23 \\
\hline & 2014 & 233 & $8.868,92$ & 15,4 & 16,95 & 56,06 & 39,11 & 0,11 & 0,07 \\
\hline & 2015 & 264 & $9.851,53$ & 18,44 & 36,55 & 177,57 & 141,02 & 0,21 & 0,14 \\
\hline & 2016 & 237 & $7.719,93$ & 19,17 & 85,01 & 299,19 & 214,18 & 0,56 & 0,28 \\
\hline \multirow{7}{*}{ 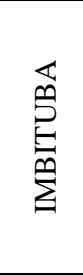 } & 2010 & 63 & $1.226,98$ & 4,75 & 6,43 & 9,15 & 2,72 & 0,41 & 0,46 \\
\hline & 2011 & 86 & $1.868,32$ & 7,05 & 9,93 & 17,62 & 7,69 & 0,53 & 1,23 \\
\hline & 2012 & 63 & $1.549,21$ & 5,3 & 6,73 & 13,78 & 7,06 & 0,53 & 0,35 \\
\hline & 2013 & 103 & $2.106,54$ & 9,94 & 15,07 & 32,3 & 17,23 & 1,26 & 0,72 \\
\hline & 2014 & 113 & $2.560,59$ & 11,26 & 17,15 & 34,99 & 17,84 & 1,21 & 0,61 \\
\hline & 2015 & 105 & $2.650,71$ & 9,62 & 18,02 & 25,95 & 7,94 & 1,62 & 0,6 \\
\hline & 2016 & 157 & $4.153,89$ & 16,89 & 31,25 & 62,39 & 31,15 & 1,64 & 1,19 \\
\hline \multirow{7}{*}{ 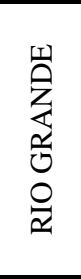 } & 2010 & 658 & $6.077,76$ & 24,85 & 42,4 & 57,21 & 14,81 & 6,75 & 6,43 \\
\hline & 2011 & 781 & $8.125,49$ & 32,13 & 52,67 & 89,21 & 36,54 & 8,99 & 5,74 \\
\hline & 2012 & 685 & $7.181,56$ & 29,78 & 50,78 & 99,13 & 48,35 & 7,72 & 6,6 \\
\hline & 2013 & 782 & $9.576,05$ & 34,22 & 64,86 & 175,99 & 111,13 & 10,4 & 7,85 \\
\hline & 2014 & 839 & $10.613,85$ & 37,92 & 71,09 & 153,8 & 82,71 & 10,07 & 6,72 \\
\hline & 2015 & 576 & $10.746,66$ & 26,13 & 58,34 & 333,77 & 275,44 & 8,43 & 5,47 \\
\hline & 2016 & 595 & $9.914,07$ & 26,36 & 80,4 & 285,81 & 205,41 & 8,41 & 6,44 \\
\hline \multirow{7}{*}{ 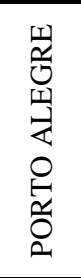 } & 2010 & 208 & 942,17 & 9,67 & 13,33 & 13,36 & 0,03 & 1,1 & 1,01 \\
\hline & 2011 & 138 & 774,74 & 5,99 & 9,4 & 9,41 & 0,02 & 0,53 & 1,02 \\
\hline & 2012 & 178 & 898,8 & 7,3 & 13,17 & 13,22 & 0,05 & 1,13 & 0,94 \\
\hline & 2013 & 183 & 997,08 & 9,27 & 16,28 & 16,58 & 0,3 & 2,21 & 2,05 \\
\hline & 2014 & 177 & $1.022,65$ & 8,48 & 13,79 & 13,88 & 0,09 & 1,62 & 1,53 \\
\hline & 2015 & 132 & 938,56 & 7,53 & 14,53 & 14,63 & 0,11 & 1,7 & 1,82 \\
\hline & 2016 & 166 & $1.048,71$ & 8,72 & 23,62 & 24,03 & 0,42 & 3,13 & 3,41 \\
\hline \multirow{7}{*}{ 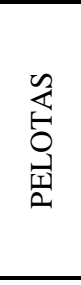 } & 2010 & 13 & 32,27 & 0,41 & 0,66 & 0,67 & 0,01 & 0,18 & 0,07 \\
\hline & 2011 & 10 & 24,9 & 0,3 & 0,4 & 0,43 & 0,02 & 0,04 & 0,07 \\
\hline & 2012 & 6 & 13,33 & 0,17 & 0,23 & 0,23 & 0 & 0,01 & 0,05 \\
\hline & 2013 & 9 & 18,55 & 0,26 & 0,4 & 0,44 & 0,04 & 0,05 & 0,09 \\
\hline & 2014 & 19 & 44,93 & 0,57 & 1,05 & 1,26 & 0,21 & 0,31 & 0,17 \\
\hline & 2015 & 18 & 49,23 & 0,6 & 1,06 & 1,07 & 0,01 & 0,27 & 0,19 \\
\hline & 2016 & 6 & 13,79 & 0,16 & 0,29 & 0,3 & 0 & 0,08 & 0,05 \\
\hline \multicolumn{2}{|c|}{ TOTAL } & 14.645 & 343.876 & 963 & 2.113 & 13.513 & 11.400 & 121 & 114 \\
\hline
\end{tabular}

Fonte: Dados da Pesquisa

A definição do tempo de operação das cargas se baseou nas especificações da Agência Nacional de Transportes Aquaviários - Antaq (2017), descritas no Quadro 1.

\section{Quadro 1 - Descrição das variáveis do modelo DEA.}

\begin{tabular}{|c|l|}
\hline Variável & \multicolumn{1}{c|}{ Descrição } \\
\hline $\begin{array}{c}\text { Tempo de } \\
\text { operação da carga }\end{array}$ & $\begin{array}{l}\text { Corresponde à divisão do tempo de operação em horas (diferença entre a data/hora final } \\
\text { de operação e a data/hora de início da operação) e o número de embarcaçães. }\end{array}$ \\
\hline $\begin{array}{c}\text { Tempo médio } \\
\text { atracado da carga }\end{array}$ & $\begin{array}{l}\text { Corresponde à divisão do tempo total atracado em horas (diferença entre a data/hora de } \\
\text { desatracação e a data/hora de atracação da embarcação no porto/instalação) e o número } \\
\text { de embarcações por porto/instalação portuária, terminal, berço ou grupo de carga. }\end{array}$ \\
\hline $\begin{array}{c}\text { Tempo de estadia } \\
\text { da carga }\end{array}$ & $\begin{array}{l}\text { Corresponde ao tempo de estadia em horas (diferença entre a data/hora da desatracação e } \\
\text { a data /hora de atracação). }\end{array}$ \\
\hline $\begin{array}{c}\text { Tempo de espera } \\
\text { para atracar }\end{array}$ & $\begin{array}{l}\text { Corresponde à divisão do tempo de espera em horas (diferença entre a data/hora da } \\
\text { atracação e a data /hora da chegada à área de fundeio) e o número de embarcações por } \\
\text { porto/instalação portuária, terminal, berço ou grupo de carga. }\end{array}$ \\
\hline $\begin{array}{c}\text { Tempo ocioso de } \\
\text { espera para iniciar } \\
\text { a operação }\end{array}$ & $\begin{array}{l}\text { Corresponde à divisão do tempo de espera em horas (diferença entre a data/hora de } \\
\text { início da operação e a data /hora da atracação) e o número de embarcações por } \\
\text { porto/instalação portuária, terminal, berço ou grupo de carga. }\end{array}$ \\
\hline
\end{tabular}




\begin{tabular}{|c|l|}
\hline Variável & \multicolumn{1}{c|}{ Descrição } \\
\hline $\begin{array}{c}\text { Tempo ocioso de } \\
\text { espera para } \\
\text { desatracar }\end{array}$ & $\begin{array}{l}\text { Corresponde à divisão do tempo de desatracação em horas (diferença entre a data/hora } \\
\text { do final da operação e a data/hora de desatracação) e o número de embarcações por } \\
\text { porto/instalação portuária, terminal, berço ou grupo de carga. }\end{array}$ \\
\hline
\end{tabular}

Fonte: Antaq (2017)

O modelo inicial se configurou em 8 variáveis e 7 DMUs e suas nomenclaturas estão descritas no Quadro 2. Para simular o modelo, utilizou-se o Sistema Integrado de Apoio à Decisão SIAD, versão 3 desenvolvido por Meza et al. (2005).

No entanto, este modelo apresentou o problema da baixa capacidade de ordenamento de eficiência, característico do método DEA. A primeira distorção, diz respeito à proporcionalidade entre o número de variáveis e DMUs. Conforme Nunamaker (1985) e Bowlin (1998), as quantidade de DMUs devem ser, pelo menos, três vezes maior do que a soma das variáveis.

Quadro 2 - Nomenclatura para identificar os portos e as variáveis do modelo DEA.

\begin{tabular}{|c|c|c|c|c|}
\hline Portos & SIGLA & & Variáveis & SIGLA \\
\hline Unidades de Tomada de Decisão & DMU & Output & Quantidade de Atracações (un) & NAVI \\
\hline Porto de Antonina & ANTO & & Peso da Carga Bruta (t) & CARG \\
\hline Porto de Paranaguá & PARA & & Tempo de Operação da Carga (h) & OPER \\
\hline Porto de São Francisco do Sul & SFSU & & Tempo Atracado da Carga (h) & ATRA \\
\hline Porto de Imbituba & IMBI & Inputs & Tempo de Estadia da Carga (h) & ESTA \\
\hline Porto do Rio Grande & RGDE & & Tempo de Espera para Atracar (h) & ESPE \\
\hline Porto de Porto Alegre & PTAL & & Tempo de Espera Iniciar Operação (h) & TEIO \\
\hline Porto de Pelotas & PELO & & Tempo de Espera Desatracar (h) & TEDE \\
\hline
\end{tabular}

Fonte: Elaborado pelos autores.

Por isso, com base em Angulo Meza et al. (2007), o número de DMUs do modelo foi ampliado e se considerou como DMU cada um dos anos da série temporal recortada (2010 a 2016). Dessa forma, o modelo DEA preliminar ficou constituído por 7 inputs, 1 output e 49 DMUs. No entanto, ocorreu dificuldade para analisar a evolução da eficiência ao longo dos anos. Nesse sentido, um modelo alternativo foi elaborado, com base na redução do número de variáveis. Para isso, realizou-se a redução de fatores pela técnica de Análise dos Componentes Principais (ACP), que permite manter a variabilidade das variáveis originais. O programa computacional utilizado foi o SPSS/IBM, versão 24.

Para provar que a matriz de dados originais cumpre com os requisitos mínimos para realizar a ACP, aplicou-se o teste de Kaiser-Meyer-Olkin (KMO) e para rejeitar a hipótese nula, pela qual a matriz de correlação poderia ser uma matriz identidade, se efetuou a Prova de Esfericidade de Bartlett (normalidade multivariada), observado o $p$-valor menor do que 0,05. Finalmente, o índice do determinante da matriz de correlação mostrou adequação, com valor menor de 0,00011. Assim, os testes comprovaram que a matriz original cumpriu os requisitos mínimos para realizar a ACP. A partir dela, se determinou as variáveis componentes do modelo DEA, composto por 2 inputs, 1 output e 7 DMUs, cumprindo os requisitos de proporcionalidade do método.

\section{ANÁLISE E DISCUSSÕES}

A aplicação do método ACP mostrou, por meio da Análise de Correlação de Pearson, a existência de associação entre as variáveis, ou seja, se constatou o problema de multicolinearidade no modelo. $\mathrm{O}$ teste do Fator de Inflação da Variância (VIF) revelou valores entre 25,393 a 44,512, considerados elevados. 
O propósito do método ACP é desenvolver um modelo parcimonioso para fazer substituição do conjunto original de variáveis fortemente correlacionadas por outro menor, com baixa ou nenhuma correlação. Para isso, excluiu-se a variável "Estadia" que se mostrou menos correlacionada com o conjunto de dados. Os testes de adequação amostral resultou em KMO igual 0,703, indicando que a porcentagem da variância dos indicadores é comum, com nível de significância de 5\%, determinado pelo teste de Esfericidade de Bartlett, o que implica em rejeitar que a matriz de correlação seria uma matriz identidade. Ainda, o determinante da matriz de correlação resultou em 0,00000545. Esses resultados mostraram que os requisitos mínimos para ser aplicada a análise de componentes principais foram cumpridos.

Os indicadores da variância total explicada mostrou dois fatores que conservam $93,3 \%$ da variabilidade total, com autovalor de 4,448 para o componente " 1 " e 1,148 para o componente " 2 ". O primeiro fator explica $74,1 \%$ da variabilidade total e o segundo $19,1 \%$. Os quatro componentes restantes apresentaram autovalor menor do que um. As somas de rotação de cargas ao quadrado indicaram que o primeiro componente explica $55,6 \%$ da variabilidade total e o segundo $37,7 \%$.

A matriz de componentes rotados refletiu na formação de dois agrupamentos, constituídos por itens de maior afinidade entre si. O componente 1 foi constituído por: tempo de espera para desatracar (TEDE); tempo de espera para iniciar operação (TEIO); tempo atracado da carga (ATRA) e tempo de espera para atracar (ESPE). Nesse agrupamento as variáveis estão relacionadas com o tempo em que o navio passa no porto, desde a sua chegada até a saída do cais, o que parece adequado denominá-lo como permanência no porto (PERM).

O componente 1 apresentou indicadores com cargas fatoriais altas e o maior percentual de variância explicada $(74,1 \%)$, o que significou a permanência no porto ser um fator bastante significativo para a gestão logística portuária. Os valores positivos das cargas fatoriais do componente 1, apontados para a mesma direção, demonstra que quando um fator apresentar tendência de elevar o grau da sua importância, os demais também terão o mesmo comportamento. De fato, o aumento da permanência dos navios no porto ocorre em função dos tempos adicionais transcorridos para atracar, de espera para iniciar as operações com as cargas e desatracar.

O componente 2, constituído pelo peso da carga bruta e tempo de operação, foi denominado de "carregamentos efetuados" ou "CARRE", que compôs 19,1\% de variância explicada, com os vetores positivos. O resultado demonstra que, se o peso da carga aumentar, o mesmo ocorrerá com o tempo de operação. De fato, esse fenômeno é comum de ser observado nas atividades do cais.

Portanto, as variáveis que influenciam a eficiência dos portos se agrupam em dois fatores, que representam a permanência e os carregamentos dos navios nos portos. Essa constatação explica a causa de alguns portos serem mais eficientes do que outros.

Tabela 2 - Eficiência padrão dos portos do Sul do Brasil, 2010 a 2016.

\begin{tabular}{cccccccc}
\hline DMU & 2010 & 2011 & 2012 & 2013 & 2014 & 2015 & 2016 \\
\hline PARA & 1,000 & 1,000 & 1,000 & 1,000 & 1,000 & 1,000 & 1,000 \\
ANTO & 0,087 & 0,491 & 0,461 & 0,563 & 0,548 & 0,348 & 0,309 \\
SFSU & 0,615 & 1,000 & 0,994 & 1,000 & 1,000 & 1,000 & 1,000 \\
IMBI & 0,303 & 0,503 & 0,354 & 0,563 & 0,638 & 0,795 & 1,000 \\
RGDE & 1,000 & 1,000 & 1,000 & 1,000 & 1,000 & 1,000 & 1,000 \\
PTAL & 1,000 & 0,807 & 1,000 & 1,000 & 1,000 & 1,000 & 0,619 \\
PELO & 0,063 & 0,058 & 0,034 & 0,049 & 0,107 & 0,136 & 0,038 \\
\hline
\end{tabular}

Fonte: Dados da pesquisa. 
A visão otimista de eficiência técnica relativa, medida pelo DEA padrão, constante na Tabela 2, mostrou que os portos de Paranaguá e do Rio Grande alcançaram a fronteira ótima de eficiência em relação à atividade logística de transbordo de granéis sólidos na área do cais em todo o período analisado. Também, os portos de São Francisco do Sul e de Porto Alegre foram $100 \%$ eficientes na maioria dos anos.

Os piores indicadores de desempenho foram observados no Porto de Pelotas, em que índices variaram de 0,034 a 0,136. O Porto de Antonina também não conseguiu alcançar indicadores satisfatórios, pois manteve a média de $40 \%$ de eficiência no período.

O DEA padrão mostrou que, de maneira geral, no período entre 2010 a 2016 os portos do Sul do Brasil foram eficientes em relação à atividade logística de transbordo de granéis sólidos. O resultado corrobora com os argumentos de Sousa Júnior et al. (2013), pois os portos que apresentaram eficiência são de grande porte.

Uma vantagem do modelo DEA é mostrar superdimensionamentos de recursos nas DMUs, como o caso de uma unidade permanecer na região viável de produção, mesmo depois de se reduzir inputs ou aumentar outpus. Por isso, foram calculadas as diferenças entre o que os portos desempenharam e os alvos que deveriam ser atingidos para o alcance da eficiência. O resultado (Tabela 3) mostrou não haver folgas nos Portos de Paranaguá e do Rio Grande, cujos alvos de eficiência foram alcançados. O mesmo não ocorreu com a maior parte dos portos, por demonstrar necessidade de aumentar o número de atracações para ser eficiente.

Tabela 3 - Alvos para o alcance da eficiência nos portos do Sul do Brasil, 2010 a 2016.

\begin{tabular}{cccccccc}
\hline DMU & 2010 & 2011 & 2012 & 2013 & 2014 & 2015 & 2016 \\
\hline PARA & - & - & - & - & - & - & - \\
ANTO & 190 & 87 & 96 & 80 & 80 & 86 & 121 \\
SFSU & 80 & 1 & - & - & - & - & - \\
IMBI & 145 & 85 & 115 & 80 & 64 & 27 & - \\
RGDE & - & - & - & - & - & - & - \\
PTAL & - & 33 & - & - & - & - & 103 \\
PELO & 195 & 161 & 172 & 174 & 158 & 114 & 151 \\
\hline
\end{tabular}

Fonte: Dados da pesquisa.

Segundo Rios (2005), as referências de cada unidade ineficiente (benchmarks) são determinadas pela projeção das DMUs ineficientes na fronteira de eficiência. O Porto de Porto Alegre foi referência para quatro unidades no período de 2010 a 2012. Nos anos seguintes, foi benchmark para os portos de Antonina, Imbituba e Pelotas, exceto em 2016, Em 2011, Porto de São Francisco do Sul se destacou como referência.

O DEA padrão revelou a presença de pesos zerados para alguns inputs e outputs em algumas DMUs. Para alcançar a eficiência, nos anos de 2010 a 2013 o Porto de Paranaguá desconsiderou o fator PERM e de 2014 a 2016 zerou o fator CARRE. O Porto do Rio Grande zerou o fator PERM entre 2011 e 2015 e CARRE no ano de 2016. A maior ocorrência de pesos zerados foi para o fator PERM, em 2014 e 2015. Por considerar PERM e CARRE os fatores extraídos pela ACP, não considera-los implica em distorções dos resultados.

Segundo Soares de Mello et al. (2005), no modelo DEA/BCC, uma DMU pode ser chamada de eficiente por default ou à partida, que significa ter obtido o menor valor de um determinado input ou maior valor em relação ao output. Para contrapor o DEA padrão e verificar se alguma DMU tenha apresentado "falsa eficiência", Entani, Maeda e Tanaka (2002) orientam o cálculo da fronteira invertida, que considera a troca dos inputs pelos outputs do modelo original de cada DMU e indica quais DMUs são ineficientes. 
Tabela 4 - Fronteira invertida para os portos ineficientes do Sul do Brasil, 2010 a 2016.

\begin{tabular}{cccccccc}
\hline DMU & 2010 & 2011 & 2012 & 2013 & 2014 & 2015 & 2016 \\
\hline PARA & $\mathbf{1 , 0 0 0}$ & $\mathbf{1 , 0 0 0}$ & $\mathbf{1 , 0 0 0}$ & $\mathbf{1 , 0 0 0}$ & $\mathbf{1 , 0 0 0}$ & $\mathbf{1 , 0 0 0}$ & $\mathbf{1 , 0 0 0}$ \\
ANTO & 0,722 & 0,119 & 0,073 & 0,087 & 0,196 & 0,391 & 0,196 \\
SFSU & 0,102 & 0,058 & 0,034 & 0,248 & 0,342 & 0,495 & $\mathbf{1 , 0 0 0}$ \\
IMBI & 0,206 & 0,116 & 0,095 & 0,087 & 0,168 & 0,171 & 0,038 \\
RGDE & $\mathbf{1 , 0 0 0}$ & $\mathbf{1 , 0 0 0}$ & $\mathbf{1 , 0 0 0}$ & $\mathbf{1 , 0 0 0}$ & $\mathbf{1 , 0 0 0}$ & 0,822 & 0,199 \\
PTAL & 0,063 & 0,072 & 0,034 & 0,049 & 0,107 & 0,136 & 0,208 \\
PELO & $\mathbf{1 , 0 0 0}$ & $\mathbf{1 , 0 0 0}$ & $\mathbf{1 , 0 0 0}$ & $\mathbf{1 , 0 0 0}$ & $\mathbf{1 , 0 0 0}$ & $\mathbf{1 , 0 0 0}$ & $\mathbf{1 , 0 0 0}$ \\
\hline
\end{tabular}

Fonte: Dados da pesquisa

A aplicação da fronteira invertida mostrou que o Porto de Paranaguá apresentou "falsa eficiência" (Tabela 4), pois foi $100 \%$ eficiente no DEA padrão e também alcançou a fronteira de ineficiência. O mesmo ocorreu no Porto do Rio Grande, nos anos de 2010 a 2014, e no Porto de São Francisco do Sul, em 2016. Isso mostra que esses portos atribuíram valores favoráveis aos inputs ou output para o alcance da fronteira ótima nos referidos períodos.

Para melhor discriminação das DMUs, o cálculo do índice de eficiência composta normalizada foi aplicado e os resultados (Tabela 5) reforçaram aqueles obtidos pela fronteira invertida. Dessa maneira pode-se concluir que os Portos de Paranaguá, Rio Grande e Pelotas não foram eficientes na atividade de transbordo de granéis sólidos na faixa do cais, no período de 2010 a 2016.

Tabela 5 - Eficiência composta normalizada dos portos do Sul do Brasil, 2010 a 2016.

\begin{tabular}{cccccccc}
\hline DMU & 2010 & 2011 & 2012 & 2013 & 2014 & 2015 & 2016 \\
\hline PARA & 0,516 & 0,515 & 0,509 & 0,513 & 0,528 & 0,537 & 0,510 \\
ANTO & 0,188 & 0,707 & 0,706 & 0,756 & 0,714 & 0,514 & 0,568 \\
SFSU & 0,781 & 1,000 & 0,997 & 0,898 & 0,876 & 0,807 & 0,510 \\
IMBI & 0,566 & 0,714 & 0,640 & 0,756 & 0,777 & 0,871 & 1,000 \\
RGDE & 0,516 & 0,515 & 0,509 & 0,513 & 0,528 & 0,632 & 0,918 \\
PTAL & 1,000 & 0,893 & 1,000 & 1,000 & 1,000 & 1,000 & 0,719 \\
PELO & 0,032 & 0,030 & 0,017 & 0,025 & 0,057 & 0,073 & 0,019 \\
\hline
\end{tabular}

Fonte: Dados da pesquisa

A análise mostrou eficiência pura para o Porto de Porto Alegre nos anos de 2010, 2012, 2013, 2014 e 2015 e para o Porto de São Francisco do Sul em 2011 e Porto de Imbituba em 2016. O Porto de São Francisco do Sul, apesar de ter alcançado $100 \%$ de eficiência pura somente no ano de 2011, apresentou altos índices de eficiência. Em suma, as DMUs com eficiência pura representaram 14\% do total, o que significa uma visão menos otimista do que a demonstrada pelo DEA padrão, que totalizou $18 \%$.

Portanto, constatou-se que os portos com $100 \%$ de eficiência pura são aqueles que obtiveram o máximo número de atracações por hora de operação, com o menor uso dos inputs (tempo de fluxo) e o Porto de Porto Alegre obteve a melhor produtividade do fator CARRE entre as DMUs.

\section{CONCLUSÃO}

O método DEA - BBC possibilitou considerar situações de eficiência com variação de escala, sem assumir proporcionalidade entre inputs e outputs e a Análise dos Componentes Principais (ACP) contribuiu de maneira significativa para atender ao requisito de proporcionalidade exigido pelo DEA, além de contornar o problema da presença de outliers.

Os resultados do DEA padrão corroboraram com o pressuposto de que os portos eficientes são aqueles capazes de produzir mais embarques e desembarques de granéis 
sólidos, sem o emprego de unidades adicionais dos recursos disponíveis. No entanto, a análise da fronteira invertida mostrou existir portos com "falsa eficiência.

O tempo de permanência (PERM) determinou a deficiência técnica dos portos e, por isso, foi considerado um importante componente dos custos logísticos. O Porto de Porto Alegre mostrou mais eficiência por apresentar menores índices do fator PERM, equivalente a 4,9\% da média realizada pelo Porto de Paranaguá, $13 \%$ em relação ao Porto de Antonina, $19 \%$ do Porto de São Francisco do Sul e 36\% dos portos do Rio Grande e de Imbituba. Ainda, esse resultado se deve à maior produtividade na relação entre os inputs (PERM e CARRE) e output (NAVI).

Além da falta de agilidade nas entregas, fatores macroeconômicos também causaram ineficiência nos portos. Nos dois últimos anos de análise o Brasil vinha sofrendo uma crise econômica e se verificou perdas de eficiência na maioria das DMUs.

Espera-se que os resultados desta pesquisa possam servir de parâmetro no processo de tomada de decisão sobre operações portuárias, além de ser possível apresentar a comparação de eficiência relativa ao tempo de entrega das cargas dos portos do Sul do Brasil, considerado fator relevante para estabelecer e/ou manter níveis de confiabilidade no atendimento dos usuários ou agentes portuários.

Devido à complexidade das operações portuárias, salienta-se que não era intuito desta pesquisa tratar de todas as atividades dos portos. Sugere-se, portanto, ampliar a análise para as demais atividades e incorporar variáveis de infraestrutura e ambiente institucional. Além disso, estender a pesquisa para portos de outras regiões e países, cujos aspectos culturais e econômicos sejam similares.

\section{REFERÊNCIAS BIBLIOGRÁFICAS}

[1] Angulo Meza, L.; Biondi Neto, L., Soares DE Mello, J. C. C. B.; Gomes, E. G. \& Coelho, P. H. G. (2003). SIAD - Sistema Integrado de Apoio à Decisão: Uma Implementação Computacional de Modelos de Análise Envoltória de Dados. Relatórios de Pesquisa em Engenharia de Produção, 3(20). Niterói: Universidade Federal Fluminense - Mestrado em Engenharia de Produção.

[2] Angulo Meza, L.; Soares de Mello, J. C. C. B.; Gomes, E. G. \& Fernandes, A. J. S. (2007). Selecção de variáveis em DEA aplicada a uma análise do mercado de energia eléctrica. Investigação Operacional, 27, 21-36.

[3] ANTAQ (2017). Sistemas de Informações Gerenciais (SIG). Gerência de estudos e desempenho portuário. Indicadores de desempenho operacional. 2010 a 2016. Disponível em: <http://www.antaq.gov.br/sistemas/sig/AreaTrabalho.asp>. Acesso em: 30 mai 2017.

[4] APPA (2016). Administração dos Portos de Paranaguá e Antonina. Caderno de resultados da Administração dos Portos de Paranaguá e Antonina: 2011-2015. Paranaguá: APPA.

[5] APSFS (2015). Administração dos Portos de São Francisco do Sul. Estatísticas portuárias 2015. Disponível em:<http://www.apsfs.sc.gov.br/wpcontent/uploads/2014/12/Dezembro-2015.pdf>. Acesso em: 24 jun. 2016

[6] Ballou, R. H. (2006). Gerenciamento da cadeia de suprimentos. Planejamento, organização e logística empresarial. 5. ed. Porto Alegre: Bookman. 
[7] Banker, R. D.; Charnes, A.\& Cooper, W. (1984). Some models for estimating technical and scale Inefficiencies in Data Envelopment Analysis, Management Science, 30, 1078-1092.

[8] Barros, C. P. (2003). The measurement of efficiency of Portuguese sea port authorities with DEA. International Journal of Transport Economics, 30(3), 335-354.

[9] Beamon, B. M. (1999). Measuring supply chain performance. International Journal of Operations \& Production Management, 9(3), 275-292.

[10] Bowersox, D. J. \& Closs, D. J. (2001). Logística empresarial: O processo de integração da cadeia de suprimento. São Paulo: Atlas.

[11] Bowlin, W. F. (1998). Measuring performance: an introduction to Data Envelopment Analysis (DEA). Journal of Cost Analysis, 14(2), 3-27.

[12] Brasil (2013). Lei n. 12.815, de 05 de junho de 2013. Dispõe sobre a exploração direta e indireta pela União de portos e instalações portuárias e sobre as atividades desempenhadas pelos operadores portuários. Brasília, 05 Jun. 2013. Disponível em: <http://www.planalto.gov.br/ccivil_03/_ato2011-2014/2013/Lei/L12815.htm>. Acesso em: 11 Mai. 2016.

[13] Brasil (2017). Ministério da Indústria, Comércio Exterior e Serviços. Balança comercial brasileira: Janeiro a dezembro de 2016. Produto por fator agregado acumulado. Disponível em: <http://www.mdic.gov.br/comercio-exterior/estatisticasde-comercio-exterior/balanca-comercial-brasileira-acumulado-do-ano>. Acesso em: 21 jul 2017.

[14] Brito, R. P., \& Brito, L. A. L. (2012). Vantagem competitiva, criação de valor e seus efeitos sobre o desempenho. RAE, São Paulo, 52(1), 070-084.

[15] Castillo-Manzano, J. I., González-Laxe, F., \& López-Valpuesta, L. (2013). Intermodal connections at Spanish ports and their role in capturing hinterland traffic. Ocean $e$ Coastal Management, 86, 1-12.

[16] Chang, Y. T., Lee, S. Y., \& Tongzon, J.L. (2008). Port selection factors by shipping lines: different perspectives berween trunk liners and feeder service providers. Marine Policy, 32(6), 877-885.

[17] Charnes, A.; Cooper, W. \& Rhodes, E. (1978). Measuring the efficiency of decision making units, European Journal of Operational Research, 2, 429-444.

[18] CONAB (2016). Companhia Nacional de Abastecimento. Levantamento de safra. Séries históricas. Soja Brasil - Safras 1976/77 a 2015/16. Disponível em: <http://www.conab.gov.br>. Acesso em: 23 mai. 2016.

[19] Corrêa, H. L.; Corrêa, C. A. (2004). Administração da produção e operações. Manufatura e serviços: uma abordagem estratégica. São Paulo: Atlas.

[20] Coto-Millan, P., Baños-Pino, J., \& Rodriguez-Alvarez, A. (2000) Economic efficiency in Spanish ports: some empirical evidence. Maritime Policy \& Management, 27(2), 169-174.

[21] CSCMP (2013). Council of Supply Chain Management Professionals. Supply Chain Management. Terms and glossary. August 2013. Disponível em: <https://cscmp.org/supply-chain-management-definitions>. Acesso em: 02 mai. 2016. 
[22] Cullinane, K., \& Wang, T. (2006). Data envelopment analysis (DEA) and improving container port efficiency. Research in Transportation Economics, 17, 517-566.

[23] Entani, T., Maeda, Y. \& Tanaka, H. (2002). Dual Models of Interval DEA and its extensions to interval data. European Journal of Operational Research, 136(1), 32-45.

[24] Farrel, M. J. (1957). The measurement of productive efficiency. Journal of the Royal Statistic Society, Series A, 120(3), 253-290.

[25] Fontes, O. H. P. M. (2006). Avaliação da eficiência portuária através de uma modelagem DEA. Dissertação (Mestrado em Engenharia de Produção). Universidade Federal Fluminense, Niterói.

[26] Guerreiro, A. S. (2006). Análise da eficiência em empresas de comércio eletrônico usando técnicas da análise envoltória de dados. Dissertação (mestrado em Engenharia Industrial) da Pontifícia Universidade Católica do Rio de Janeiro, Rio de Janeiro.

[27] Haguenauer, L. (1989). Competitividade: conceitos e medidas. Uma resenha da bibliografia recente com ênfase no caso brasileiro. Texto para Discussão n. 211. Rio de Janeiro: IEI/UFRJ.

[28] Heskett, J. L (1994). Controlling customer logistics service. International Journal of Physical Distribution and Logistics Management, 24(4), 4-10.

[29] Huggins, R.; Izushi, H. \& Thompson, P. (2013). Journal of Centrum Cathedra: The Business and Economics Research Journal, 6(2), 155-172.

[30] Koopmans, T. C. (1951). Activity analysis of production and allocation. In: Proceedings of a conference, John Wiley and Sons, New York.

[31] Kupfer, D. (1991). Padrões de concorrência e competitividade. Texto de Discussão n. 265. Rio de Janeiro: IEI/UFRJ.

[32] Martins, K. V. (2015). Análise da eficiência relativa de portos multipropósito. Dissertação (Mestrado em Engenharia Civil). Universidade Federal do Espírito Santo, Vitória.

[33] MDIC (2016). Ministério da Indústria, Comércio Exterior e Serviços. Comércio Exterior. Séries Históricas. Grupos de produtos: exportação. Jan. 1997- abr. 2016. Disponível em: <www.mdic.gov.br>. Acesso em: 03 mai. 2016.

[34] Menegazzo, L. R., \& Fachinello, A. L. (2014). Análise de nível de eficiência dos portos brasileiros. Revista de Economia, 40(3), 173-197.

[35] Meza, L. A.; Biondi, L. N.; Soares de Mello, J. C. C. B. S. \& Gomes, E. G. (2005). ISYDS - Integrated System for Decision Support (SIAD - Sistema Integrado de Apoio à Decisão): a software package for data envelopment analysis model. Pesquisa Operacional, 25(3), 493-503.

[36] Nunamaker, T. R. (1985). Using Data Envelopment Analysis to measure the efficiency of non-profit organizations: A critical evaluation. Managerial and Decision Economics, 6(1), 50-58.

[37] Nwanosike, F., T.; Nicoleta, S. \&Warnock-Smith, D. (2012). An evaluation of Nigerian ports post-concession performance. In: Proceedings of the 17th Annual Logistics Research Network Conference. Chartered Institute of Logistics and Transport. 
[38] OMC (2013). Organização Mundial do Comércio. Trends in international trade: Factors shaping the future of world. Trade world trade report 2013. Disponível em: <https://www.wto.org/english/res_e/booksp_e/wtr13-2b_e.pdf>. Acesso em 15 set 2017.

[39] Peixoto, M. G. M. (2013). Avaliação da eficiência operacional de terminais intermodais da região sudeste na cadeia logística de grãos por análise envoltória de dados. Dissertação (Mestrado em Engenharia de Produção). Universidade Federal de São Carlos, São Carlos.

[40] Pires, J. S. (2016). A eficiência técnica dos portos e terminais públicos e privados brasileiros marítimos no período de 2010 a 2014. Dissertação (Mestrado em Transportes). Departamento de Engenharia Civil e ambiental da Universidade de Brasília. Brasília.

[41] Porter, M. (1990). The competitive advantage of nations. Harvard Business Review, 73-93.

[42] Rios, L. R. (2005). Medindo a eficiência relativa das operações dos terminais de containeres do Mercosul. Dissertação (Mestrado em Administração). Universidade Federal do Rio Grande do Sul, Porto Alegre.

[43] Rios, C. O. (2015). Mensuração de eficiência: um novo exame aplicado aos portos públicos brasileiros. Dissertação (Mestrado em Economia do Desenvolvimento). Universidade Católica do Rio Grande do Sul, Porto Alegre.

[44] Roll, Y., \& Hayuth, Y. (1993). Port performance comparison applying data envelopment analysis (DEA). Maritime Policy \& Management, 20(2), 153-161.

[45] Samuelson, P. A. \& Nordhaus, W. D. (2012). Economia. 19. ed. Porto Alegre: CMGH.

[46] SEP/PR (2012). Secretaria dos Portos da Presidência da República. Operação técnica para apoio à SEP/PR no planejamento do Setor portuário brasileiro e na implantação dos projetos de inteligência logística portuária. Plano mestre Porto de São Francisco do Sul. Florianópolis.

[47] SEP/PR (2012a). Secretaria dos Portos da Presidência da República. Operação técnica para apoio à SEP/PR no planejamento do Setor portuário brasileiro e na implantação dos projetos de inteligência logística portuária. Plano mestre Porto de Imbituba. Florianópolis.

[48] SEP/PR (2013). Secretaria dos Portos da Presidência da República. Cooperação técnica para apoio à SEP/PR no planejamento do setor portuário brasileiro e na implantação de projetos de inteligência logística portuária. Plano mestre do Porto de Paranaguá. Florianópolis.

[49] SEP/PR (2013a). Secretaria dos Portos da Presidência da República. Cooperação técnica para apoio à SEP/PR no planejamento do setor portuário brasileiro e na implantação de projetos de inteligência logística portuária. Plano mestre do Porto de Porto Alegre. Florianópolis.

[50] SEP/PR (2013b). Secretaria dos Portos da Presidência da República. Cooperação técnica para apoio à SEP/PR no planejamento do setor portuário brasileiro e na implantação de projetos de inteligência logística portuária. Plano mestre do Porto de Pelotas. Florianópolis. 
[51] SEP/PR (2013c). Secretaria dos Portos da Presidência da República. Cooperação técnica para apoio à SEP/PR no planejamento do setor portuário brasileiro e na implantação de projetos de inteligência logística portuária. Plano mestre do Porto de Rio Grande. Florianópolis.

[52] SEP/PR (2013d). Secretaria dos Portos da Presidência da República. Cooperação técnica para apoio à SEP/PR no planejamento do setor portuário brasileiro e na implantação de projetos de inteligência logística portuária. Plano mestre do Porto de Rio Grande. Florianópolis.

[53] Slack, N., Chambers, S., \& Johnston, R. (1997). Administração da Produção. 2. ed. São Paulo: Atlas.

[54] Soares de Mello, J. C. C. B. et al. (2005). Curso de análise de envoltória de dados. In: XXXVII Simpósio Brasileiro de Pesquisa Operacional, SBPO, Gramado (RS), 27 a 30 set. 2005.

[55] Sousa Júnior, J. N. C., Nobre Júnior, E. F., Prata, B. A., \& Mello, J. C. C. B. S. (2013). Avaliação da eficiência dos portos utilizando análise envoltória de dados: estudo de caso dos portos da região nordeste do Brasil. Journal of Transport Literature, Manaus, 7(4), 75-10.

[56] SUPRG (2016). Superintendência do Porto de Rio Grande. Autoridade portuária. Disponível em: <http://www.portoriogrande.com.br>. Acesso em: 23 mai. 2016.

[57] Vergara, S. C. (2004). Projetos e relatórios de pesquisa em administração. 5. ed. São Paulo: Ed. Atlas.

[58] Vieira, G. B. B. (2013). Modelo de governança aplicado a cadeias logísticoportuárias. Tese (Doutorado em Engenharia de Produção). Escola de Engenharia da Universidade Federal do Rio Grande do Sul, Porto Alegre.

[59] Vieira, G. B. B. \& Kliemann Neto, F. J. (2016). Taxonomy for the classification of container ports: A contribution to port governance. Revista Espacios, 37(3), 23-39.

[60] Wanke, P. F., \& Hijjar, M. F. (2009). Exportadores brasileiros: estudo exploratório das percepções sobre a qualidade da infraestrutura logística. Produção, 19(1) 143-162.

[61] Wanke, P. F.; Barbastefano, R. G. \& Hijjar, M. F. (2011). Determinants of efficiency at major brazilian Port terminals. Transport Reviews, 31(5), 653-677.

[62] Wanke, P. F., \& Barros, C. P. (2015). Public-private partnerships and scale efficiency in Brazilian ports: Evidence from two-stage DEA analysis. Socio-Economic Planning Sciences, 51, 13-22. 